\title{
INTER-OBSERVER AGREEMENT IN CLINICAL OPTIC DISC MEASUREMENT USING A MODIFIED 60 D LENS
}

\author{
R. S. HASLETT ${ }^{1}$, M. BATTERBURY ${ }^{1}$, M. CUYPERS ${ }^{2}$ and R. L. COOPER ${ }^{2}$ \\ Liverpool and Perth, Western Australia
}

\begin{abstract}
SUMMARY
Purpose: To assess the inter-observer agreement of the measurement of optic disc dimensions by two observers using a modified 60 dioptre (D) fundus examination lens.

Method: The vertical disc and cup diameters of 29 eyes were measured by two independent observers using a $60 \mathrm{D}$ lens modified by incorporation of a 0.1 millimetre scale graticule. The vertical cup/disc ratio was calculated. Inter-observer agreement was assessed by calculation of the inter-observer differences and by the weighted kappa statistic.

Results: The two observers showed good agreement for the measurement of disc diameter (mean difference -0.04 ; range $-0.04,0.2$ ) and for cup diameter (mean difference -0.03 ; range $0.3,0.2)$. Closer agreement for the vertical cup/disc ratio was achieved (mean kappa $0.96 ; 95 \%$ confidence limits $0.90,1.0)$. The $95 \%$ confidence limit for the mean inter-observer difference in cup/disc ratio was 0.11 , suggesting that a change of $>0.1$ in the assessment of the cup/disc ratio by this technique is significant at the $5 \%$ level.

Conclusion: High inter-observer agreement of optic disc measurement can be achieved with this technique. The method has the potential to improve the clinical evaluation of the optic disc and the precision and accuracy of the clinical measurement of other fundal structures.
\end{abstract}

Assessment of the optic disc, combined with tonometry and visual field analysis, forms a fundamental part of the evaluation of the patient with glaucoma and of the glaucoma suspect. Assessment of the optic disc should be accurate and repeatable so that change can be detected with confidence. However, clinical assessment, which includes study of disc size

From: ${ }^{1}$ University of Liverpool, Liverpool, UK; ${ }^{2}$ Lions Eye Institute, Perth, Western Australia.

Correspondence to: M. Batterbury, FRCOphth, Department of Medicine, University of Liverpool, PO Box 147, Liverpool L69 3BX, UK. Tel: +44 (0151) 706 4912. Fax: +44 01517065802. and neuroretinal rim contour and colour, is subjective. The vertical cup/disc (C/D) ratio $^{1}$ has been used both in diagnosis and in follow-up, in conjunction with other features of glaucomatous damage. Unfortunately the usefulness of the $C / D$ ratio is limited by inter- and intra-observer errors. ${ }^{2,3}$ Even amongst glaucoma experts there is often disagreement. ${ }^{4,5}$ Several techniques have been proposed to improve the accuracy of optic disc assessment by clinical, photographic and automated image analysis systems. $^{6-9}$ The scanning laser ophthalmoscope is the latest instrument to be developed for this purpose, but it is not yet in widespread clinical use. ${ }^{10-12} \mathrm{~A}$ recent report suggests that despite the limitations of clinical assessment of the optic disc it compares favourably with some of the currently available quantitative techniques. ${ }^{13}$

Most clinical ophthalmologists do not have access to archived photographs of the optic disc or to image analysers, so there is still a need for a repeatable clinical examination technique. In this study the vertical diameters of the optic disc and the cup were measured by two independent observers using a 60 dioptre (D) lens, modified by incorporation of a graticule. The results were used to calculate the C/D ratio. The aim of the study was to assess the interobserver agreement of this technique.

\section{METHOD}

\section{The Modified 60 D lens (Fig. 1)}

A standard $60 \mathrm{D}$ (Volk) indirect fundus examination lens for slit lamp use was removed from its mount and incorporated into one end of a barrel. The barrel was in two halves, united by a screw thread. A 0.1 millimetre scale graticule (Peake Instruments) was fixed at the other end of the barrel. Stereo-biomicroscopy is performed by holding the modified lens close to the subject's eye in the conventional way. After focusing on the fundal structure (the optic disc in this case), a focused image of the millimetre scale is 
superimposed on the fundal image by rotating the anterior portion of the barrel with respect to the fixed posterior portion at the screw thread. Fixation of the subject's fellow eye assists in correct alignment of images of the fundus and of the scale.

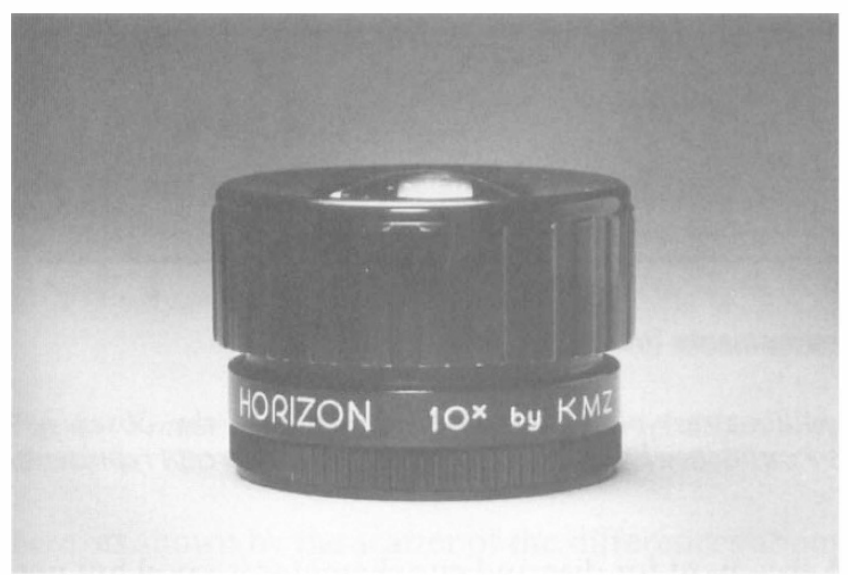

(a)

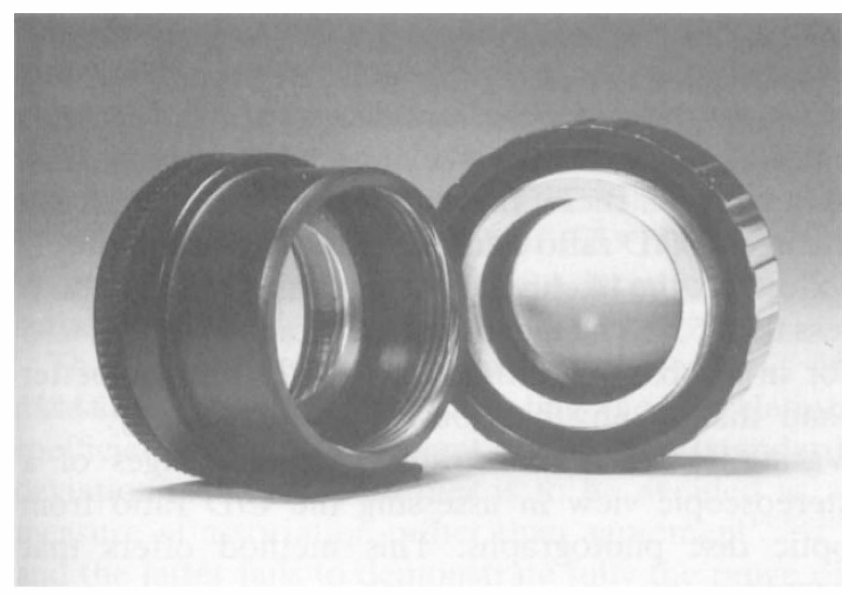

(b)

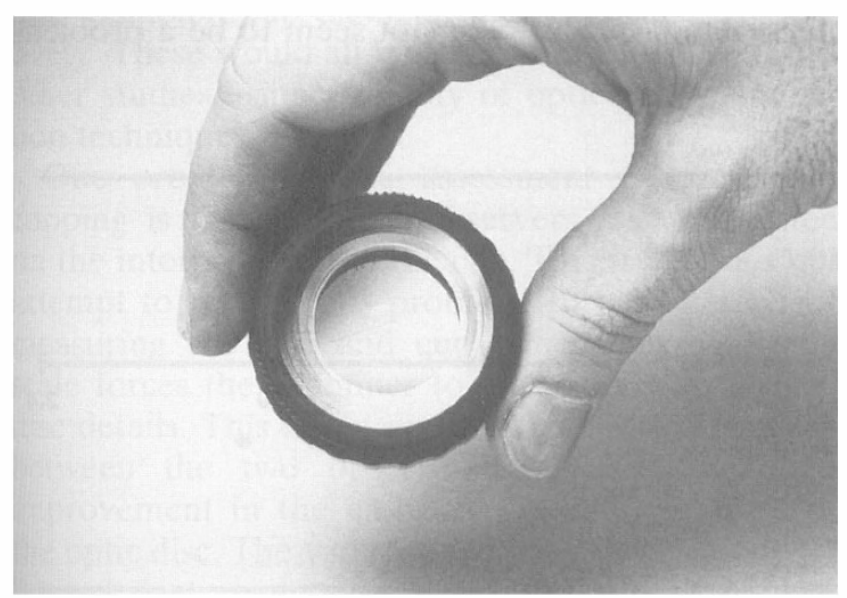

(c)

Fig. 1. (a) The modified $60 \mathrm{D}$ lens. (b) The two parts of the modified $60 \mathrm{D}$ lens screw together. The distance between the two lenses can be varied. (c) The modified lens is held in the conventional way.

\section{Subjects}

Twenty-nine eyes of 15 patients were examined consecutively from a group who had undergone pupillary dilation in the course of their attendance at a general ophthalmic clinic. The observers were unaware of any clinical information. Each observer independently examined each optic disc and recorded the vertical disc and cup measurements to the nearest $0.1 \mathrm{~mm}$. The vertical $\mathrm{C} / \mathrm{D}$ ratio was calculated from these values. Units were not ascribed to the measurements. The diameter of the optic disc was defined by the inner border of the scleral canal, but in order to simulate the normal clinical situation and in recognition of the difficulties of interpreting definitions of the cup/rim boundary, the cup/rim boundary was not strictly defined. However, change in contour was judged more important than change in colour. The same slit lamp was used for each examination.

\section{Statistical Analysis}

Two methods were used to assess inter-observer agreement:

1. Calculation of the difference between the measurements made by each observer for each disc and calculation of the mean of those two measurements. ${ }^{14}$ From these, the $95 \%$ confidence limits for the inter-observer difference were calculated, as $1.96 \times$ the standard deviation of the differences. ${ }^{14,15}$ This analysis can be presented as a plot of the interobserver differences against their means, plus $95 \%$ confidence limits. The bias, that is the tendency for one observer to underestimate a measurement compared with the other observer, was assessed by comparing the mean of the differences against zero (single $t$-test) ${ }^{15}$

2. The weighted kappa test is an appropriate test for categorical data ${ }^{16}$ and has been used before to assess the agreement of the vertical cup/disc ratio. ${ }^{3}$ Clinical importance is improved by deriving a weighted score. Following calculation of the difference in the $C / D$ ratio as measured by the two observers for each disc, the weighted kappa score was derived as 1 minus the difference.

\section{Vertical Disc Diameter}

\section{RESULTS}

The mean vertical disc diameter was 2.13 (range 1.75, 2.80 ). The mean difference was -0.04 (range -0.4 , 0.2 ; standard deviation $0.16 ; 95 \%$ confidence limit 0.31 ). This mean (the bias) was not significant $(p=0.21)$. Fig. 2 is the plot of the inter-observer differences against their means.

\section{Vertical Cup Diameter}

The mean vertical cup diameter was 0.95 (range 0 , 2.05). The mean difference was -0.03 (range -0.3 , 


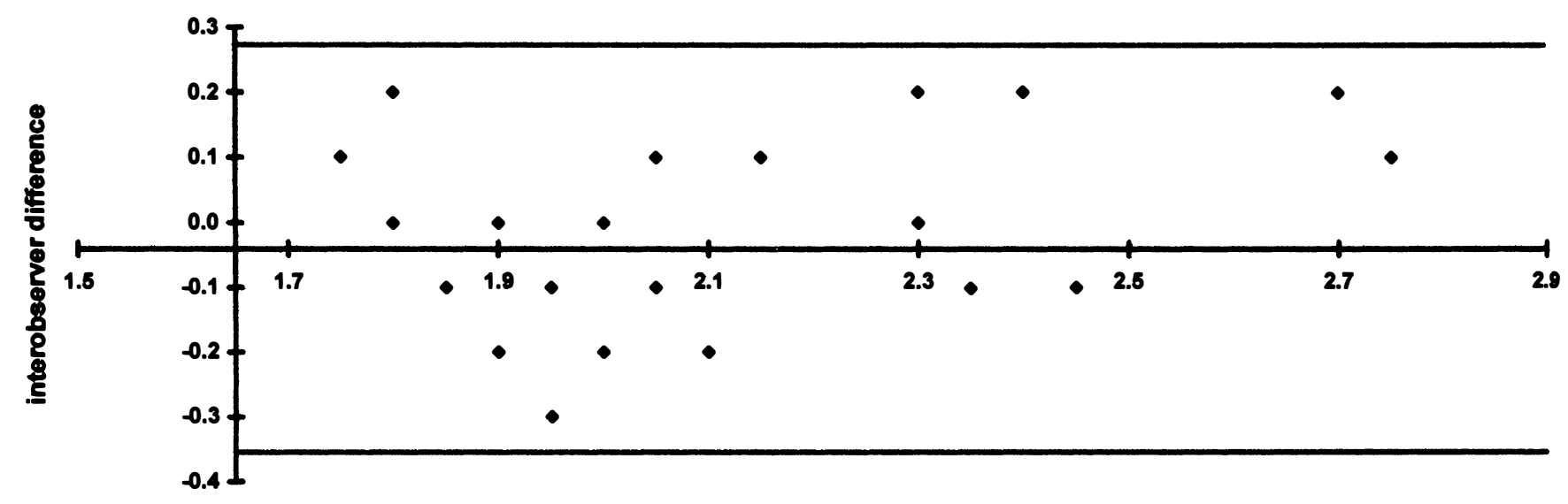

mean of observer measurements $(\mathrm{mm})$

Fig. 2. Scatter diagram showing inter-observer difference (in millimetres) plotted against the mean of the observers' measurement of the vertical disc diameter (in millimetres). The $95 \%$ confidence limits are plotted. The $\mathrm{x}$, $\mathrm{y}$ intercept represents the mean inter-observer difference (the bias).

0.2 ; standard deviation $0.12 ; 95 \%$ confidence limit $0.24)$. The bias was not significant ( $p=0.84)$. Fig. 3 is the plot of the inter-observer differences against their means.

\section{Cup/Disc Ratio}

The mean C/D ratio was 0.45 (range $0,0.92$ ). The mean difference in $\mathrm{C} / \mathrm{D}$ ratio was 0.0 (range -0.1 , 0.13 ; standard deviation 0.06 ; $95 \%$ confidence limit 0.11 ). The mean kappa statistic was 0.96 (standard deviation 0.03 ; $95 \%$ confidence limit 0.063 ). Agreement to within 0.1 was achieved in all but one eye. Fig. 4 is the plot of the inter-observer differences against their means.

\section{DISCUSSION}

The purpose of this study was to test inter-observer agreement in the measurement of the vertical optic disc and cup diameter and of the vertical $C / D$ ratio using a modified $60 \mathrm{D}$ fundus examination lens.
Agreement for disc and cup diameter is good but not exceptional ( $95 \%$ confidence limits of 0.31 and 0.24 on means of 2.13 and 0.95 , respectively). However, agreement for $\mathrm{C} / \mathrm{D}$ ratio is exceptionally good, with $95 \%$ confidence limit of 0.11 . This suggests that although the two observers disagreed on the actual cup and disc diameter, they agreed on the relationship between them. In addition, it suggests that if a change in $\mathrm{C} / \mathrm{D}$ ratio of more than 0.1 is found during follow-up, the likelihood of this change being false is less than $5 \%$. The mean weighted kappa score of 0.95 for inter-observer difference in $\mathrm{C} / \mathrm{D}$ ratio is better than that of any previously published study. ${ }^{2,3,5,17}$ Varma et al..$^{5}$ demonstrated the advantages of a stereoscopic view in assessing the $\mathrm{C} / \mathrm{D}$ ratio from optic disc photographs. This method offers that benefit.

A potential problem in any measurement methodology is variation in error across the range of measurements. This does not seem to be a problem

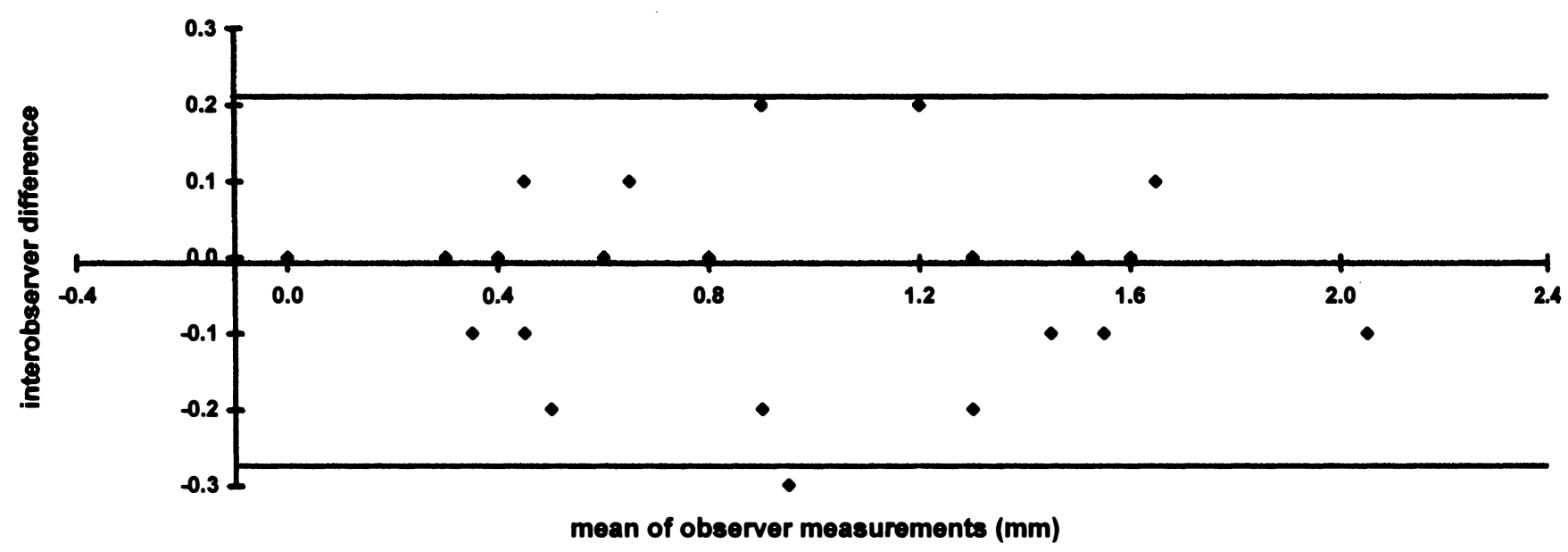

Fig. 3. Scatter diagram showing inter-observer difference (in millimetres) plotted against the mean of the observers' measurement of the vertical cup diameter (in millimetres). The $95 \%$ confidence intervals are plotted. The $\mathrm{x}$, $\mathrm{y}$ intercept represents the mean inter-observer difference (the bias). 


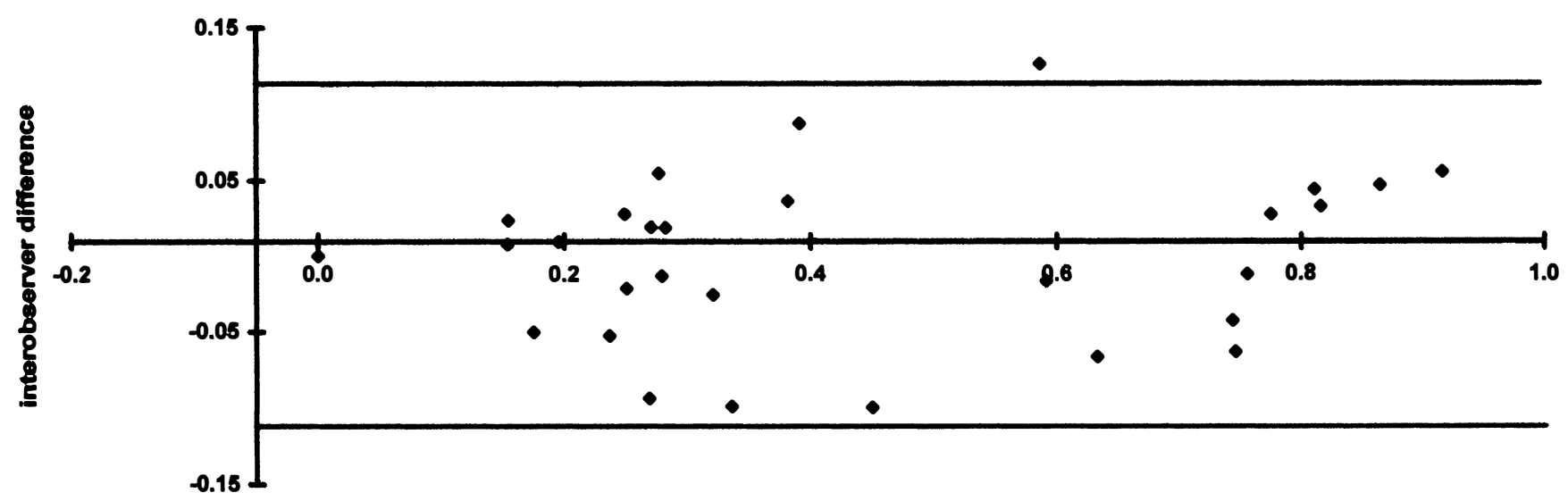

mean of observer measurements

Fig. 4. Scatter diagram showing inter-observer difference plotted against the mean of the observers' measurement of the cup/ disc ratio. The $95 \%$ confidence limits are plotted. The $\mathrm{x}, \mathrm{y}$ intercept represents the mean inter-observer difference (the bias).

here, as shown by the scatter of the differences about the mean difference in Figs. 2-4. This was confirmed by linear regression (not charted). In particular, the agreement in C/D ratio was not worse for those discs in which change may be most difficult to detect and yet most important in clinical terms, i.e. in the $0.3-0.8$ range. However the $\mathrm{C} / \mathrm{D}$ ratio is being used as a surrogate for neuroretinal rim measurement and the relationship between $\mathrm{C} / \mathrm{D}$ ratio and neuroretinal rim is non-linear. ${ }^{18}$ At high values of $\mathrm{C} / \mathrm{D}$ ratio a small change in $\mathrm{C} / \mathrm{D}$ ratio represents a large change in neuroretinal rim.

There are several techniques for the analysis of measurement agreement, including the correlation coefficient and the coefficient of variation (standard deviation/mean). The former is to be avoided as a measure of association rather than agreement ${ }^{14.15 .19}$ and the latter fails to demonstrate fully the range of disagreement. Further analysis of our data reveals coefficients of variation of $4.4 \%, 8 \%$ and $8.2 \%$ for disc diameter, cup diameter and $\mathrm{C} / \mathrm{D}$ ratio, respectively. These would all be acceptable compared with other studies using a variety of optic disc examination techniques. $8,7,20-23$

One problem in the assessment of optic disc cupping is that different observers may not agree on the interpretation of the cup. This study does not attempt to resolve this problem. In fact, the act of measuring the disc and cup dimensions against a scale forces the examiner to pay more attention to disc details. This might explain the good agreement between the two observers and might lead to improvement in the elevation of other features of the optic disc. The value of assessing the $\mathrm{C} / \mathrm{D}$ ratio is limited since many optic discs have a large physiological cup with a normal neuroretinal rim area. Larger discs have larger physiological cups. ${ }^{24}$ Hence it is important to estimate the size of the disc as well as the relationship between neuroretinal rim area and cup area. Estimation of optic disc size can be achieved by indirect biomicroscopy, using the variable height of the slip lamp beam projected onto the disc through a $90 \mathrm{D}$ lens. ${ }^{25}$ This method, analysed by correlation for intra-observer reproducibility alone, was advocated to identify rapidly the small disc with an abnormal though small cup and the large disc with a physiologically normal but large cup. Spencer and Vernon ${ }^{26}$ used a $78 \mathrm{D}$ lens and found good intra-observer reproducibility for disc size. Neither study estimated C/D ratio. This study is the first to report inter-observer agreement for cup diameter and $\mathrm{C} / \mathrm{D}$ ratio as well as for disc diameter using a simple clinical approach. Montgomery ${ }^{27}$ proposed a neuroretinal rim index to take into account the normal variations in disc and cup size. This could be calculated using our measurement technique.

Accuracy (closeness of the measurements to the true value) must also be considered. Ruben ${ }^{25}$ used planimetric data to calculate a nomogram for derivation of actual values from the slit beam height. However, the clinical usefulness of this has been questioned. ${ }^{28}$ The $78 \mathrm{D}$ lens was found to overestimate disc size by $0.12 \mathrm{~mm}$ when compared with planimetry, and $30 \%$ of values differed from the planimetric result by $0.2 \mathrm{~mm}$ or more. ${ }^{26}$ Jonas and Papastathopoulos $^{29}$ measured the disc parameters using a Goldmann lens and slit beam height and found that this underestimated disc size when compared with planimetry. Image analysers require data about refraction, or keratometry and axial length, for calculation of actual values. Measurement of several parameters, each with its own measurement error, for the purpose of calculating a final measurement may increase the likelihood that the final value is not accurate, if measurement errors are large. It therefore becomes increasingly important that measurement error be minimised the greater the number of parameters that are being measured. 
Given that the optical system of the emmetropic eye has a total refractive power of approximately 60 $\mathrm{D},{ }^{30}$ measurements made through a $60 \mathrm{D}$ lens will be accurate. In refractive ametropia there is no change in image size. There will be no change in image size between examination sessions with the development of cataract-induced myopia. In axial ametropia, $1 \mathrm{D}$ of ametropia alters image size by $1.66 \%$ in a linear manner. Emmetropic eyes of power other than $60 \mathrm{D}$ will produce images of different size. ${ }^{30}$ Thus in many eyes, the method described here will yield absolute values of the size of a fundus structure. That would be a definite advantage over other techniques. In other eyes, with knowledge of keratometry and refraction, absolute values could be derived from a correction factor.

C. O'Brien (personal communication) measured disc diameters with the scanning laser ophthalmoscope (HRT, Heidelberg Instruments) and by slit lamp biomicroscopy with the $60 \mathrm{D}$ lens. Regression of the HRT measurement on the $60 \mathrm{D}$ lens measurement produced a formula of $y=0.85 x+$ 0.06 , indicating that the $60 \mathrm{D}$ lens magnifies the image by approximately $17 \%$ compared with the HRT. Using this formula, our mean vertical disc diameter is 1.87 , similar to that quoted by Jonas and Papastathopoulos ${ }^{29}$ from photographs corrected for magnification and similar to O'Brien's results.

Associated with ametropia is displacement of the fundus image. ${ }^{30}$ This modification of the $60 \mathrm{D}$ lens permits the simultaneous visualisation of focused images of the fundus structure and of the measurement scale. This is an improvement over other systems in which the graticule is at a fixed distance from the lens, particularly in ametropia and in emmetropia in situations in which the lens and the eye cannot be correctly positioned in relation to each other.

Even if absolute values cannot be measured, it is still useful to be able to make reproducible measurements for within-subject longitudinal study. In addition, it is an advantage to be able to complete a clinical measurement at the time of an examination, avoiding problems associated with the acquisition of digitised or photographic images which may not be apparent until later, such as poor quality, movement or equipment failure. Selection bias, towards certain types and sizes of disc, has been avoided, particularly with regard to $C / D$ ratio (range $0,0.92$ ).

As a fundus lens, the modified lens is not difficult to use. Focusing one-handed requires some practice and could be improved by reducing friction of the screw thread. Subject eye movement was a problem, but was helped by fixation of the fellow eye on a suitable target, such as the fixation target on the slit lamp. Use of this target also helped in superimposing the millimetre scale on the disc. Awkward reflections from the scale occurred and might be reduced by multicoating. The scale was orientated vertically, but can be rotated into any position. Thus disc measurement at other meridians can be made. Measurement was achieved in eyes with various degrees of cataract and through intraocular lens implants.

This study indicates that precise (reproducible) optic disc measurements can be made using a $60 \mathrm{D}$ lens modified by incorporation of a focusable millimetre scale. Using this lens it may for the first time be possible to quantify optic disc parameters for follow-up examination and to detect real, clinically important change. The conventional $60 \mathrm{D}$ lens is already in use by optometrists and technicians. Use of the modified lens by such personnel might improve the quality of screening programmes and follow-up visits. The modified lens can also be used in the measurement of other fundal structures, such as choroidal naevi, and in the assessment of macular disease, by measuring the distance between the pathology and the centre of the macula. The fact that the graticule can be rotated easily simplifies the measurement of any structure in any meridian at the posterior pole. We plan further studies to investigate these potential applications.

Key words: Inter-observer agreement, Optic disc, Measurement, Clinical.

\section{REFERENCES}

1. Armaly MF, Sayegh RE. The cup/disc ratio: the findings of tonography and tonometry in the normal eye. Arch Ophthalmol 1969;82:191-6.

2. Kahn HA, Leibowitz H, Ganley JP, Kini M, Colton T, Nickerson R, Dawber TR. Standardising diagnostic procedures. Am J Ophthalmol 1975;79:768-75.

3. Abrams LS, Scott IU, Spaeth G, Quigley HA, Varma R. Agreement among optometrists, ophthalmologists and residents in evaluating the optic disc for glaucoma. Ophthalmology 1994;101:1662-7.

4. Lichter PR. Variability of expert observers in evaluating the optic disc. Trans Am Ophthalmol Soc 1976;74:532-72.

5. Varma R, Steinmann WC, Scott IU. Expert agreement in evaluating the optic disc for glaucoma. Ophthalmology 1992;99:215-21.

6. Cooper RL, Adler VA, Constable IJ. Measurement vs judgement of cup:disc ratios: statistical evaluation of intra-observer and inter-observer error. Glaucoma 1982;4:169-76.

7. Caprioli J, Klingbeil U, Sears M, Pope B. Reproducibility of optic disc measurements with computerised analysis of stereoscopic video images. Arch Ophthalmol 1986;104:1035-9.

8. Shields MB, Martone JF, Shelton AR, Ollie AR, MacMillan J. Reproducibility of topographic measurements with the optic nerve head analyser. Am J Ophthalmol 1987;104:581-6.

9. Montgomery DMI. Measurement of optic disc and neuroretinal rim areas in normal and glaucomatous eyes: a new clinical method. Ophthalmology 1991;98:50-9. 
10. Mikelberg FS, Wijsman K, Schulzer M. Reproducibility of topographic parameters obtained with the Heidelberg Retina Tomograph. J Glaucoma 1993;2:101-3.

11. Spencer AF, Sadiq SA, Pawson P, Vernon SA. Vertical optic disc diameter: discrepancy between planimetric and SLO measurements. Invest Ophthalmol Vis Sci 1995;36:796-803.

12. Mikelberg FS, Parfitt CM, Swindale NV, Graham SL, Drance SM, Gosine R. Ability of the Heidelberg Retina Tomograph to detect early glaucomatous visual field loss. J Glaucoma 1995;4:242-7.

13. O'Connor DJ, Zeyen T, Caprioli J. Comparison of methods to detect glaucomatous optic nerve damage. Ophthalmology 1993;100:1498-503.

14. Bland JM, Altman DG. Statistical methods for assessing agreement between two methods of clinical measurement. Lancet 1986;1:307-10.

15. Zadnik K, Mutti DO, Adams AJ. The repeatability of measurements of the ocular components. Invest Ophthalmol Vis Sci 1992;33:2325-33.

16. Fleiss JL. Statistical methods for rates and proportions. New York: Wiley, 1981:212-35.

17. Tielsch JM, Katz J, Quigley HA, Miller NR, Sommer A. Intra-observer and inter-observer agreement in measurement in optic disc characteristics. Ophthalmology 1988;95:350-6.

18. Montgomery DMI. Clinical disc biometry in early glaucoma. Ophthalmology 1993;100:52-6.

19. Zadnik K, Mutti DO. Statistical analysis for method comparison data. Arch Ophthalmol 1993;111:582-3.
20. Jonas JB, Gusek GC, Naumann GOH. Optic disc morphometry in chronic primary open angle glaucoma. I. Morphometric intrapapillary characteristics. Graefes Arch Clin Exp Ophthalmol 1988;226:522-30.

21. Varma R, Steinmann WC, Spaeth GL, Wilson RP. Variability in digital analysis of optic disc topography. Graefes Arch Clin Exp Ophthalmol 1988;226:435-42.

22. Mikelberg FS, Douglas GR, Schulzer M, Cornsweet TN, Wijsman K. Reliability of optic disc topographic measurements recorded with a video-ophthalmolograph. Am J Ophthalmol 1984;98:98-102.

23. Klein BEK, Magli YL, Richie KA, Moss SE, Meuer SM, Klein R. Quantitation of optic disc cupping. Ophthalmology 1985;92:1654-6.

24. Montgomery DMI. Measurement of optic disc and neuroretinal rim areas in normal and glaucomatous eyes: a new clinical method. Ophthalmology 1991; 98:50-9.

25. Ruben S. Estimation of optic disc size using indirect biomicroscopy. Br J Ophthalmol 1994;78:363-4.

26. Spencer AF, Vernon SA. Optic disc measurement: a comparison of indirect ophthalmoscopic methods. Br J Ophthalmol 1995;79:910-5.

27. Montgomery DMI. Clinical disc biometry in early glaucoma. Ophthalmology 1993;100:52-6.

28. Barr DB. Estimation of optic disc size [letter]. Br J Ophthalmol 1995;79:298-9.

29. Jonas JB, Papastathopoulos K. Ophthalmoscopic measurement of the optic disc. Ophthalmology 1995; 102:1102-6.

30. Elkington JR, Frank HJ. Clinical optics, 2nd ed. Oxford: Blackwell Scientific, 1991. 